that genetic abnormalities of mitosis can produce some sub-diploid variation ${ }^{2}$. But the normal existence of such a large proportion of sub-diploid and particularly of sub-haploid cells within the same tissue would be a fact of special interest.

In order to check on this reported chromosome variation, $I$ have examined the chromosome numbers in cells of the human uterine endometrium. As further controls, the normal uterine endometrium of Rattus norvegicus (laboratory albino rat) with $2 n=42$, and of Microtus agrestis (short-tailed bank vole) with $2 n=50$, have also been cxamined. The rodent Microtus agrestis is especially favourable for somatic chromosome studies since the two sex chromosomes are considerably larger than any of the 48 autosomes and they can be readily identified during the stages of mitosis ${ }^{3}$.

In both the present and Timonen's ${ }^{1}$ observations, the cytological study was based on the use of Feulgen squashes. This technique can give satisfactory results for mammalian chromosomes ${ }^{4}$. But in making such preparations, one has to be very careful that the cells have not been damaged by the squashing. In the scoring of chromosome numbers, I have therefore taken particular care that no damaged cells were ineluded. All chromosome counts have been made with an accuracy of about 5 per cent of the chromosomes or even better. Fifty cells were scored in each of the three species, and these have shown no variations from the normal diploid chromosome numbers. In addition, it was clear from the appearance of many dividing cells which could not be so accurately counted that there is no extensive sub-diploid variation.

Some reductions in chromosome number as a result. of such mechanisms as lagging, non-disjunction, or an occasional multipolar spindle, may normally occur in some somatic cells. Multiples of the diploid number may also occur. But there is no indisputable evidence of extensive sub-diploid variation as the usual constituent of normal somatic tissues.

Leo SACeS

Department of Experimental Biology,

Weizmann Institute of Science,

Rehovoth, Israel.

${ }^{1}$ Timonen, S., and Therman, E., Nature, 168, 995 (1950). Timonen,
S., Acta Obstetr. Scand., 81, Supp. $2,1(1950)$. Therman, F., and Timonen, S., Hereditas, 37, 266 (1951).

${ }^{2}$ Sachs Leo, Heredity, 6, 157 (1952).

s Sachs, I.eo, Heredity, [7. 227 (1953)].

- Sachs, I.eo, Heredity, 6, 357 (1952).

\section{An Irreversible Choline-esterase Inhibitor in White Clover}

Is the course of a study of the decomposition products of radioactive octamethylpyrophosphoramide in white clover (Trifolium repens, strain S100) it was found incidentally that extracts from clover, whether previously treated with this compound or not, inhibited choline-esterase in vitro.

Clover samples were macerated with water, the macerate extracted with chloroform (equal volume) and the chloroform extract evaporated nearly to dryness under vacuum. Water was then introduced, the remainder of the chloroform distilled off, and the aqueous solution filtered free of the chlorophyll precipitated. The anticholine-esterase activities of the aqueous products from untreated clover were found by Michel's method', using freeze-dried plasma. The aqueous products from treated clover were evaporated under vacuum in the presence of trichloroethylene, thus obtaining chlorophyll-free solutions in this solvent of the chloroform extractables without subjecting them to a temperature above $20^{\circ} \mathrm{C}$. or to prolonged contact with water. The trichloroethylene solutions were then chromatographed on a kieselguhr column, with a water standing phase and a 30 per cent chloroform : 70 per cent trichloroethylene $(\mathrm{v} / \mathrm{v})$ moving phase. The eluent fractions were tested for anticholine-esterase activity as before.

The total anticholine-esterase activity is expressed as the volume of solution in millilitres extracted from $1 \mathrm{kgm}$. of clover inducing 50 per cent inhibition in a serum solution after incubation for $30 \mathrm{~min}$. at $25.6^{\circ} \mathrm{C}$. In these units, four untreated samples gave values of $3,640,4,550,640$ and $32^{*}$; two treated samples, 2,140 and 10,620 ; and volumes of solvents equivalent to those used in the extractions, 20-30. There are considerable variations. Most are proba.bly to be accounted for by absorption on the chlorophyll precipitated when the chloroform is removed. It was later discovered that, unless special precautions are taken, up to 80 per cent of a low concentration of octamethylpyrophosphoramide may be absorbed by the chlorophyll in this way, and that the amount absorbed is very variable. Probably the inhibitor is similarly absorbed. The starred value is, however, very much lower than the rest. The sample of clover for which this value was obtained came from another field of $S 100$ clover in a very different state of growth, and yielded little or no inhibitor.

One active sample was tested by Mr. K. P. Fellowes for in vitro activity against human and rat red cell choline-esterase, and for reversibility towards rat cells by the method, slightly modified, of Aldridge ${ }^{2}$. It was about equally active against human and rat red cells as against human plasma, and showed no signs of reversibility after washings equivalent to $6.4 \times 10^{7}$ dilution.

The inhibitor from the treated samples was eluted from the column before the octamethylpyrophosphoramide or any other phosphorus-32-containing band appeared. It therefore separates more readily than these compounds from water into the organic solvents used. Its anticholine-esterase activity was only reduced to $a$ third by the action of $N$ sodium hydroxide for $30 \mathrm{~min}$. at $15^{\circ} \mathrm{C}$, and was not observably affected by storage in water for one week.

If this compound can be absorbed by animals eating large quantities of clover, it might be expected that they would show some ill-effects. Bloat in sheep and cattle is attributed to cyanogenetic compounds produced by several varieties of clover ${ }^{3}$. It is certain that the substance we have discovered does not inhibit choline-esterase by producing cyanide, as $M / 100$ potassium cyanide only induces 20 per cent inhibition in plasma choline-esterase under our experimental conditions; but this does not rule out the possibility that the choline-esterase inhibitor is identical with one of the cyanogenetic compounds. Thus clover may in some cases produce signs of anticholine-esterase poisoning as well as cyanide poisoning. We think, therefore, that these observations may have some relevance to bloat.

$$
\text { D. F. HeAth }
$$

P. O. PARK

Pest Control, Ltd.,

Harston, Cambridge. March 9.

1 Michel, H. O., J. Lab, Clin. Med., 34, 1504 (1949).

2 Aldridge, W. N., Biochem. J., 46, 451 (1950).

${ }^{3}$ Melville, J., and Doak, B. W., N.Z. J. Sci. and Tech., 22 B, 674

(1944). "Evans, W. C., and Rees Evans, E. T., Nature, 163, 373 (1949). 